\title{
Oxygen Control For Bioreactors And In-vitro Cell Assays
}

\author{
V. Nock ${ }^{1}$, R. J. Blaikie ${ }^{1}$ and T. David ${ }^{2}$ \\ ${ }^{I}$ MacDiarmid Institute for Advanced Materials and Nanotechnology, Department of Electrical and Computer \\ Engineering, University of Canterbury, Private Bag 4800, Christchurch, New Zealand \\ ${ }^{2}$ Centre for Bioengineering, Department of Mechanical Engineering, University of Canterbury, Private Bag 4800, \\ Christchurch, New Zealand \\ volker.nock@elec.canterbury.ac.nz
}

\begin{abstract}
Dissolved oxygen (DO) is an important parameter in biomedical and cell-culture applications. Several studies have found cell survival and function to be intimately linked to oxygen concentration. Laminar flow, as observed in microfluidic devices, provides an ideal environment to manipulate and control concentration gradients. In this paper we demonstrate the first characterization of integrated fluorescence-based oxygen sensors for DO measurement within a cell-culture bioreactor device. Solid-state PtOEPK/PS sensor patterns were integrated into the PDMS-based bioreactor and calibrated for detection of DO concentration with a superimposed layer of collagen and Ishikawa human endometrial cancer cells. The sensor signal of the layer subjacent to the cells was found to follow a Stern-Volmer model and the intensity ratio was measured to $\mathrm{I}_{0} / \mathrm{I}_{100}=3.9$ after 3 days in culture. The device provides a novel tool for the control and spatially-resolved measurement of oxygen levels in cellular assays and cell-culture applications.
\end{abstract}

Keywords: Laminar flow, bioreactor, PtOEPK/PS sensor, dissolved oxygen, cell-culture.

PACS: $47.61 . \mathrm{Fg}$

\section{INTRODUCTION}

Laminar flow, as observed in microfluidic devices, allows for partial treatment of cell-cultures and individual cells with biochemical reagents ${ }^{1}$ without the need for physically separated culture chambers. This technique provides a powerful tool for studies in cell biology. Recently however, oxygen concentration, a further parameter with significant effect on cell behaviour, has started to gain increasing interest. Dissolved oxygen (DO) concentration has been found to be intimately linked to cell survival, metabolism and function ${ }^{2-5}$. These results demonstrate the requirement to precisely control and measure DO levels for an increase in relevance of in-vitro experiments in cell biology.

To address this issue we have developed a microfluidic device, shown in Fig. 1(a), which is capable of generating flow with controlled oxygen concentrations. Based on a novel sensor fabrication process $^{6}$ and modular gas exchangers, the device combines luminescence-based polymer optical oxygen sensors and laterally-tapering polydimethylsiloxane (PDMS) microfluidic channels to yield custom longitudinal ${ }^{7}$ oxygen gradients inside the bioreactor. By adding additional inlet flows, as depicted in
Fig. 1(b), the device can be further extended to provide transverse graduation through multiple parallel laminar streams of different oxygen concentrations ${ }^{8}$.

While the previous examples are useful for various applications, such as selective cell treatment ${ }^{9}$ and measurement of the coefficient of diffusion for oxygen in a perfusion media ${ }^{8}$, the final goal is to use the sensor in-situ in a sandwich configuration with cells cultured on top of the sensor to map the oxygen distribution throughout the bioreactor. Figure 1(c) shows a schematic cross-section of the device based on a glass substrate, a sensor layer, an extra-cellular matrix protein (ECM, collagen), cells and a PDMS microfluidic structure. In this paper we show initial results demonstrating the sensor function in a multilayer system with a collagen monolayer and live human cancer cells. The device, in conjunction with cell-culture, provides a novel tool for the control and spatially-resolved measurement of oxygen levels in cellular assays and cell-culture applications.

\section{EXPERIMENTAL}

The multi-stream cell-culture bioreactor chip and the integrated gas exchangers were fabricated using micro-lithography and replica-molding in PDMS. 

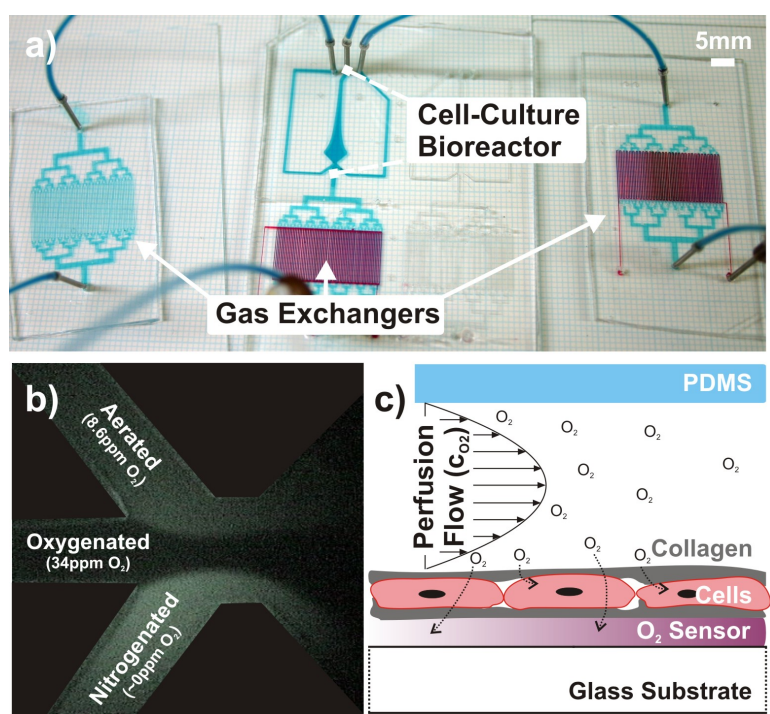

FIGURE 1. (a) Photograph of the bioreactor device with integrated PtOEPK/PS oxygen sensor layer. (b) Fluorescence intensity image showing an example of three parallel flow streams entering the bioreactor. (c) Schematic of the multilayer sandwich configuration for in-situ oxygen measurement.

Details of the fabrication process and integration of the oxygen sensor layer from a solution containing platinum (II) octaethylporphyrin ketone dye (PtOEPK, Frontier Scientific) and $7 \%(\mathrm{w} / \mathrm{v})$ polystyrene (PS, Sigma Aldrich) via soft-lithography have been described previously ${ }^{6}$. For use with cells, an additional cell-adhesion promoting layer of $0.01 \%(\mathrm{w} / \mathrm{v})$ type I collagen (rat tail, Sigma Aldrich) was deposited onto the sensor film. Typically, collagen solution was pipetted into a PDMS gasket outlining the PtOEPK sensor shape, left to incubate overnight at room temperature and rinsed with DI water before use.

\section{Cell-Culture}

Ishikawa human endometrial cancer cells were obtained from cryo-storage at $-80^{\circ} \mathrm{C}$ in a freezer or in liquid nitrogen. Cell dispersion was carried out under aseptic conditions. Preparation was performed in a laminar flow cell culture hood. All glassware used for cell preparation was autoclaved before use and together with all the solutions were sterilized with ethanol. All cells (in cryo-vial) and solutions were first pre-warmed to $37^{\circ} \mathrm{C}$ before use. Minimum Essential Medium $\alpha$ Medium ( $\alpha$ MEM) supplemented with a $10 \%$ of Fetal Bovine Serum Medium was used culture medium. Once the cells were ready, they were transferred into culture flasks $(50 \mathrm{ml})$ and $10 \mathrm{ml}$ of the $\alpha \mathrm{MEM}$ was added to each flask. The cells were then stored in an incubator $\left(37^{\circ} \mathrm{C}, 0.5 \% \mathrm{CO}_{2}\right)$ for at least 1-
2 days to allow the cells to adhere to the surface. Culture medium was changed after 1-2 days and replaced with another $10 \mathrm{ml}$ of ( $\alpha$ MEM + FBS $10 \%)$.

When cells had reached a confluent state, they were split in preparation for further use. Cultures that had become confluent were placed in the cell-culture hood, where the cell culture media was removed by aspiration. Pre-warmed $10 \mathrm{ml}$ of Phosphate Buffered Saline (10x PBS pH7.4) at $37^{\circ} \mathrm{C}$ was used to wash the cells. The PBS was then removed and the wash step was repeated twice more. Following the final aspiration of the PBS from the flask, $2.5 \%$ trypsin in PBS was added to the cells. Cells were dislodged by light tapping onto the side of the flask. The split cells were transferred into a larger culturing plate for different experiments. A small sample of a prepared cell suspension was removed, and used to perform a cell count with a haemocytometer. Cells were then centrifuged at $1500 \mathrm{rpm}$ for $5 \mathrm{~min}$ at room temperature. After that, the supernatant was aspirated and the media ( $\alpha$ MEM + FBS 10\%) was added, with the volume dependent on the cell count performed earlier, the size of the target substrate and the concentration of cells required. The substrate was then stored in the incubator for 3 days before experiment.

\section{Oxygen Measurement}

To demonstrate sensor function with cells the sensor intensity was recorded as a function of oxygen partial pressure before and after culture. In both cases, the device was exposed to air $\left(\sim 21 \% \mathrm{O}_{2}\right)$, oxygen-free nitrogen $\left(0 \% \mathrm{O}_{2}\right)$, industrial grade oxygen $\left(100 \% \mathrm{O}_{2}\right)$ at $37^{\circ} \mathrm{C}$ and the intensity change was measured using a fluorescence microscope (Eclipse 80i, Nikon), as described previously ${ }^{6}$.

\section{RESULTS AND DISCUSSION}

The PtOEPK/PS system was chosen as oxygen sensor material as it provides a highly sensitive measurement platform. Due to the solid-state nature of the sensor film substrates can be re-used, making it a low-cost alternative to dissolved fluorescence sensors. Preparations of the material are considered biocompatible and have been used to measure cellular oxygen uptake rates ${ }^{10}$ and test food packaging. ${ }^{11}$ To test the function of the sensor system under exposure to cell-culture conditions and in contact with live cells, Ishikawa human endometrial cancer cell line was chosen due to its favorable growth characteristics in culture. $^{12}$

Figure 2 shows a schematic and the dimensions of the PtOEPK/PS sensor pattern on the bottom surface of the bioreactor. Collagen was selectively deposited 

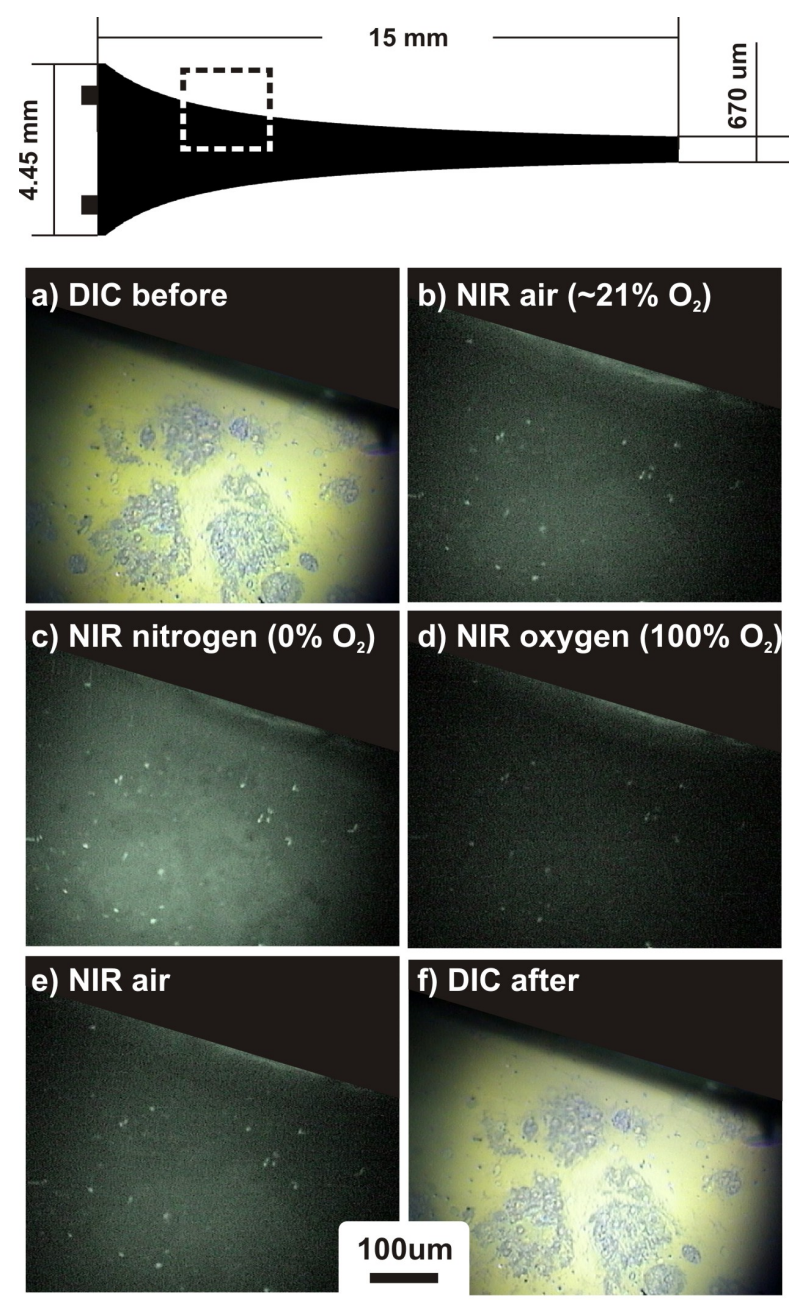

FIGURE 2. Schematic and micrographs showing a patterned $\mathrm{PtOEPK} / \mathrm{PS}$ oxygen sensor layer after 3 days in culture with type I collagen and human endometrial cancer cells. (a) DIC image of cell clusters in a region of the bioreactor indicated by the dashed square. (b) - (e) The same area as fluorescent intensity image in NIR for exposure to $21 \%, 0 \%, 100 \%$ and $21 \% \mathrm{O}_{2}$, respectively, showing the change of intensity in the underlying sensor layer. (f) DIC image after fluorescent microscopy.

onto this pattern and cells were successively seeded on top of the collagen support. A differential interference contrast (DIC) micrograph of a section of the pattern is shown in Fig. 2(a) indicating good adherence and the formation of cell clusters. Following visual inspection, the whole bioreactor was exposed to different gaseous oxygen concentrations. Images in Fig. 2(b) to (e) show fluorescent intensity of the same section of the bioreactor chamber in near-infrared (NIR) for exposure to $\sim 21 \% \mathrm{O}_{2}$ (air), $0 \% \mathrm{O}_{2}$ (pure nitrogen), $100 \% \quad \mathrm{O}_{2} \quad$ (pure oxygen) and again $\sim 21 \% \quad \mathrm{O}_{2}$, respectively. As shown by the image sequence, the sensor layer subjacent to the cells retains its ability to reversibly indicate changes in oxygen concentration via a change in fluorescence intensity. This demonstrates that the sensor can thus be used to measure the oxygen concentration at the bottom of the bioreactor. Since the measured values are produced by oxygen diffusion through the cell layer the system is directly linked to the concentration on the surface of the cell layer. Through visual inspection of the intensity images it can be further observed that the dense cellular clusters visible in Fig. 2(a) do not significantly affect the localized change of intensity in the sensor layer.

To quantify the potential influence of the cellular covering layer on sensor function, the change in intensity as a function of oxygen concentration was derived from the intensity images shown in Fig. 2(b)(e). A plot of inverse relative fluorescent intensity as a function of oxygen partial pressure for a $7 \%(\mathrm{w} / \mathrm{v})$ PtOEPK/PS sensor layer prior to and after deposition of type I collagen and seeding of human endometrial cancer cells is shown in Fig. 3. The intensity ratio $\mathrm{I}_{0} / \mathrm{I}_{100}$, with $\mathrm{I}_{0}$ being the intensity for exposure to $0 \%$ $\mathrm{O}_{2}$ and $\mathrm{I}_{100}$ the intensity for $100 \% \mathrm{O}_{2}$, for a $600 \mathrm{~nm}$ thick $7 \%(\mathrm{w} / \mathrm{v}) \mathrm{PtOEPK} / \mathrm{PS}$ layer was measured to be 8.1 .

After deposition of $\sim 5 \mu \mathrm{g} / \mathrm{cm}^{2}$ type I collagen, seeding of a layer of endometrial cancer cells and incubating the substrate, sensor and cell stack for 3 days in nutrient solution, the intensity response of the sensor still follows a linear Stern-Volmer model. The intensity ratio in the multi-layer configuration compared to the uncovered sensor layer is reduced to $\mathrm{I}_{0} / \mathrm{I}_{100}=3.9$. While this is a significant reduction compared to the initial value for an uncovered film, it is still larger than a ratio $\mathrm{I}_{0} / \mathrm{I}_{100}$ of 3 , with which a sensor is commonly considered a suitable oxygen sensing device. ${ }^{13}$

The fact that the intensity ratio remains high enough for in-situ use despite this reduction is a direct result of the sensor fabrication process used, which allows one to repeatedly deposit homogeneous films with a high intensity ratio ${ }^{6}$. In addition, the solid-state nature of the sensor significantly reduces the amount of dye needed compared to dissolved sensors and reduces the contact between the biomaterials and sensor through encapsulation in the PS matrix. Recently we have shown that a layer of the ECM protein collagen deposited directly on top of the sensor film has no discernible effect on the oxygen sensing capabilities even after an extended period of incubation. ${ }^{14}$ From this we conclude that the reduction in intensity ratio in the current configuration is mainly due to the additional cell layer covering the sensor, which limits the oxygen transport to the sensor to diffusion through the cell layer.

The initial tests with human cancer cells presented in this paper demonstrate successful sensor function 


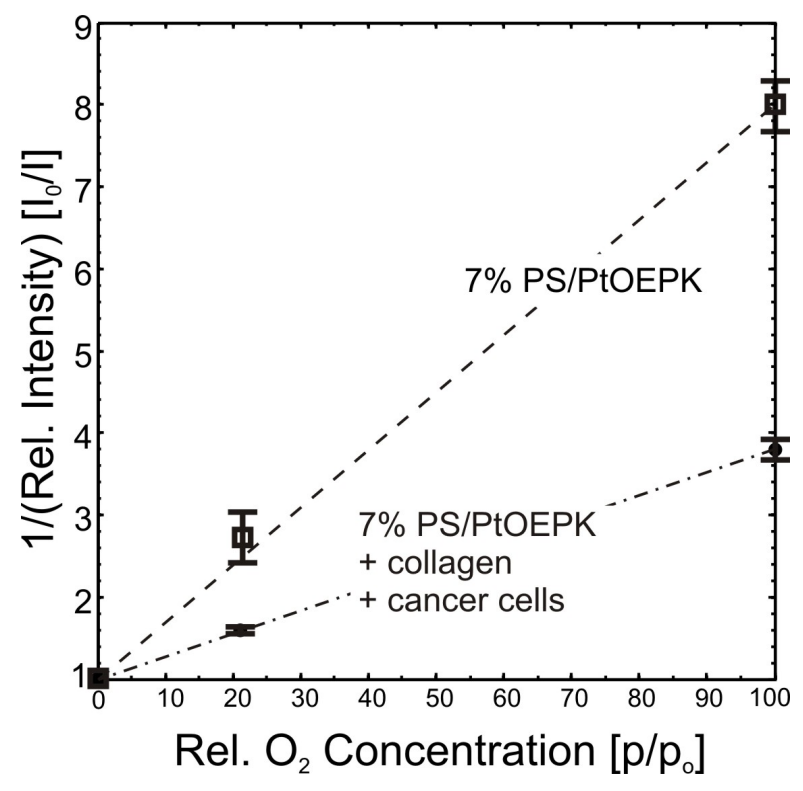

FIGURE 3. Plot of relative fluorescent intensity vs. oxygen concentration for a 7\% (w/v) PtOEPK/PS sensor layer with and without collagen and endometrial cancer cells cultured on top.

after several days in culture, thus indicating the potential for in-situ oxygen measurement with live cells. Whilst a reduction in relative fluorescence intensity was observed, the measured $\mathrm{I}_{0} / \mathrm{I}_{100}$ ratio remains adequate to make the system a candidate for use as an embedded oxygen sensor layer in cell-culture applications. Further experiments regarding sensor function and oxygen measurements in long-term cell perfusion are planned, as are investigations into the influence of the attached cells on the sensor signal.

\section{CONCLUSIONS}

We have shown the integration and application of a fluorescent dye-based oxygen sensing layer in a PDMS-based microfluidic cell-culture device. Sensor function was demonstrated for a PtOEPK/PS layer subjacent to a collagen film and a layer of human endometrial cancer cells. The intensity ratio of the layer for exposure to oxygen was measured prior to and after cell seeding. While it was found to decrease to approximately half the value of the initial film, the ratio exhibited by the covered film remains high enough to be suitable for in-situ measurements. In combination with the sensor integration and spatial oxygen control, the presented chip provides a novel tool for a multitude of applications, such as in-situ oxygen control in cell-culture bioreactors, parallelization of oxygen concentration-dependent assays and tissue engineering.

\section{ACKNOWLEDGMENTS}

The authors would like to thank Fahmi Samsuri and Prof. John Evans at the University of Otago, Christchurch School of Medicine \& Health Sciences, for providing the cancer cells and Helen Devereux and Gary Turner for technical assistance.

\section{REFERENCES}

1. S. Takayama, E. Ostuni, P. LeDuc, K. Naruse, D. E. Ingber and G. M. Whitesides, Nature 411 (6841), 10161016 (2001).

2. S. Roy, S. Khanna, A. A. Bickerstaff, S. V. Subramanian, M. Atalay, M. Bierl, S. Pendyala, D. Levy, N. Sharma, M. Venojarvi, A. Strauch, C. G. Orosz and C. K. Sen, Circ Res 92 (3), 264-271 (2003).

3. L.-L. Zhu, L.-Y. Wu, D. Yew and M. Fan, Mol. Neurobiol. 31 (1), 231-242 (2005).

4. H. Zhang and G. Semenza, J. Mol. Med. 86 (7), 739-746 (2008).

5. R. Fukuda, H. Zhang, J.-w. Kim, L. Shimoda, C. V. Dang and Gregg L. Semenza, Cell 129 (1), 111-122 (2007).

6. V. Nock, R. J. Blaikie and T. David, Lab Chip 8 (8), 1300-1307 (2008).

7. V. Nock, R. J. Blaikie and T. David, Proc. SPIE 6799, 67990Y-67910 (2007).

8. V. Nock, R. J. Blaikie and T. David, Proc. MicroTAS 1, 299-301 (2008).

9. S. Takayama, E. Ostuni, P. LeDuc, K. Naruse, D. E. Ingber and G. M. Whitesides, Chem. Biol. 10 (2), 123130 (2003).

10. C. O'Donovan, J. Hynes, D. Yashunski and D. B. Papkovsky, J. Mat. Chem. 15 (27-28), 2946-2951 (2005).

11. M. Smiddy, N. Papkovskaia, D. B. Papkovsky and J. P. Kerry, Food Res. Intern. 35 (6), 577-584 (2002).

12. M. Nishida, Human Cell 15 (3), 104-117 (2002).

13. B. D. MacCraith, C. M. McDonagh, G. O'Keeffe, E. T. Keyes, J. G. Vos, B. O'Kelly and J. F. McGilp, Analyst $118,385-388(1993)$

14. V. Nock, R. J. Blaikie and T. David, Proc. ICBME 2008 23 (2008). 